\title{
Characteristics and Identification of Sites of Chagasic Ventricular Tachycardia by Endocardial Mapping
}

\author{
Maria Zildany P. Távora, Niraj Mehta, Rose M. F. L. Silva, Fernando A. A. Gondim, \\ Vanderlei M. Hara, Angelo A. V. de Paola \\ São Paulo, SP - Brazil
}

\begin{abstract}
Objective - To study electrophysiological characteristics that enable the identification and ablation of sites of chagasic tachycardia.

Methods - Thirty-one patients with chronic Chagas' heart disease and sustained ventricular tachycardia (SVT) underwent electrophysiological study to map and ablate that arrhythmia. Fifteen patients had hemodinamically stable SVT reproducible by programmed ventricular stimulation, 9 men and 6 women with ages ranging from 37 to 67 years and ejection fraction varying from 0.17 to 0.64 . Endocardial mapping was performed during SVT in all patients. Radiofrequency $(R F)$ current was applied to sites of presystolic activity of at least $30 \mathrm{~ms}$. Entrainment was used to identify reentrant circuits. In both successful and unsuccessful sites of RF current application, electrogram and entrainment were analyzed.
\end{abstract}

Results - Entrainment was obtained during all mapped SVT. In 70.5\% of the sites we observed concealed entrainment and ventricular tachycardia termination in the first 15 seconds of RF current application. In the unsuccessful sites, significantly earlier electrical activity was seen than in the successful ones. Concealed entrainment was significantly associated with ventricular tachycardia termination. Bystander areas were not observed.

Conclusion - The reentrant mechanism was responsible for the genesis of all tachycardias. In $70.5 \%$ of the studied sites, the endocardial participation of the slow conducting zone of reentrant circuits was shown. Concealed entrainment was the main electrophysiological parameter associated with successful RF current application. There was no electrophysiological evidence of bystander regions in the mapped circuits of SVT.

Key words: chagasic cardiomyopathy, ventricular tachycardia, catheter ablation

Universidade Federal de São Paulo - Escola Paulista de Medicina

Mailing address: Maria Zildany P. Távora - EPM - Clinical Electrophysiology Department - Rua Napoleão de Barros, 593 - 04024-002 - São Paulo, SP - Brazil
Chagas' disease is one of the most important health issues in Latin America ${ }^{1}$. In endemic regions, it is the main cause of sudden death, affecting many young apparently healthy, asymptomatic or oligosymptomatic individuals, with preserved or little compromised ventricular function ${ }^{24}$.

In patients with structural heart disease, despite the great technological advances provided by electrophysiological study, ventricular tachyarrhythmias remain challenging, both in their clinical manifestations, being many times fatal in the first event, and in their therapeutic management, being generally refractory to several drug schemes. The lack of prospects, in the near future, of new more effective antiarrhythmic agents has stimulated the scientific community to use and improve new alternative nonpharmacological techniques, such as catheter ablation and implantable defibrillator.

Radiofrequency ablation (RF) is a minimally invasive treatment. In patients with sustained ventricular tachycardia (SVT) of ischemic etiology, encouraging success rates, varying from 42 to $75 \%{ }^{5-12}$, have been achieved, leading to increasing scientific interest in improving this technique. This procedure requires detailed knowledge of the arrhythmogenic substrate because the lesion produced by RF energy is localized and a precise identification of the site of origin of the tachycardia is necessary.

Electrophysiological definitions - Fractionated electrogram-electrical activity with multiple components, low amplitude, increased duration, and high frequency ${ }^{13}$; amplitude - measurement (in $\mathrm{mV}$ ) of the greatest deflection of the intracavitary electrogram (peak-to-peak) ${ }^{13}$; durationmeasurement of electrogram extent (in ms), from the first to the last electrical activity varying from the stable baseline ${ }^{13}$; presystolic activity - electrical activity detected during SVT, before any intracavitary ventricular electrogram or QRS complex onset ${ }^{14}$; entrainment - technique consisting of stimulus trains during SVT, using a cycle at least $20 \mathrm{~ms}$ less than the tachycardia cycle length, accelerating the tachycardia to the stimulated frequency. Stopping the stimulation, no change is seen in the tachycardia cycle or 
morphology ${ }^{14}$. When the stimulation is performed far from the tachycardia circuit, a change in morphology during stimulation (fusion of the QRS complex) is seen. In this situation, the first post-pacing interval equals the stimulation cycle length; entrainment with concealed fusion (concealed entrainment) - maintenance of the electrocardiographic configuration of SVT during stimulation is seen; it occurs when stimulation is performed in pathways connected to the reentrant circuit $^{9}$. This way, the depolarization wave resulting from the stimulus follows the same trajectory of the activation wave of SVT, depolarizing the ventricle from the same site and producing a QRS morphology similar to that of SVT; return cycle - is the first postpacing interval (between the last electrogram stimulated during entrainment and the next electrogram of SVT) ${ }^{9}$; stimulus to QRS interval - interval between the spike produced by stimulation and the onset of the earliest QRS complex during entrainment ${ }^{9}$; bystander areas - regions of slow conduction connected to the reentrant circuit, but not participating in it, protected by areas of anatomic blockade ${ }^{9}$; excitable gap - time interval in a cycle of frequency in which a determined tissue (atrium, ventricle or reentrant circuit) is out of the refractory period, allowing its depolarization; success of RF ablation-interruption of ventricular tachycardia in the first $15 \mathrm{~s}$ of energy delivery.

\section{Methods}

Thirty-one patients sequentially referred to our service and admitted to the Hospital São Paulo - UNIFESP, from June/94 to December/96, with chronic Chagas' heart disease (CCHD) (positive serology for Chagas' disease) were eligible for this study. They had clinical manifestation of SVT, but neither manifest heart failure or pregnancy, nor any other kind of heart or severe chronic disease. Of these, 15 patients (nine men and six women, with a mean age of 52.6 years) were selected for this study. In each patient SVT was hemodynamically stable in the electrophysiologic laboratory allowing mapping its origin site.

Except for two patients with well-tolerated incessant SVT, all the others had varied symptoms of low cardiac output (dizziness, sweating, extreme fatigue and dyspnea). Three had a syncopal phenomenon and one underwent cardiopulmonary resuscitation. Five patients were in functional class (FC) III (NYHA) and the remaining in FC I or II. All had clinical manifestation of SVT under the effect of at least one class III antiarrhythmic drug ${ }^{15}$.

Eleven patients had basal sinus rhythm, which alternated with atrial flutter in one and with incessant SVT rhythm in another. Four patients used definitive pacemakers, three of which were dual chamber pacemakers and another was a single chamber pacemaker. In the latter patient, the rhythm of the pacemaker alternated with that of incessant SVT.

The ejection fraction (EF) analyzed by left ventricular angiography varied from 0.17 to 0.64 (mean $=0.37 \pm 0.13$ ). All patients had segmental or diffuse ventricular dysfunction. Twelve patients had hypokinesia, akinesia or dyskinesia of posterobasal or inferoposterior walls, and five patients had these alterations in the apical region. Diffuse hypokinesia without segmental dysfunction of the left ventricle (LV) was evidenced in only two individuals. No patient had obstructive coronary artery disease identified by coronary angiography (tab. I).

Electrophysiological study was performed without anesthetic sedation. Initially, two punctures of the right femoral vein were performed, through which two multipolar catheters for diagnosis were introduced, with interelectrode intervals of $1 \mathrm{~cm}$. Under radioscopic vision, a catheter was placed in the subtricuspid region to record the His bundle and another in the high right atrium (HRA) to record and stimulate the atria. Later, this catheter was sequentially repositioned in the apex and in the right ventricle (RV) outflow tract for recording and stimulation.

\begin{tabular}{|c|c|c|c|c|c|c|}
\hline Pts & Name & $\begin{array}{c}\text { Age } \\
\text { (years) }\end{array}$ & Sex & Events & $\mathrm{EF}$ & $\mathrm{FC}$ \\
\hline 01 & MARP & 42 & $\mathrm{~F}$ & SVT++ & 0.37 & II \\
\hline 02 & SS & 62 & M & SVT++ & 0.64 & I \\
\hline 03 & AGG & 64 & $\mathrm{~F}$ & SVT+++ & 0.37 & III \\
\hline 04 & VB & 37 & M & SVT++++ & 0.17 & III \\
\hline 05 & IFS & 62 & $\mathrm{~F}$ & SVT+++ & 0.58 & II \\
\hline 06 & ALC & 55 & M & SVT+++ & 0.36 & I \\
\hline 07 & VAS & 49 & M & SVT++ & 0.51 & II \\
\hline 08 & JMC & 58 & M & SVT++ & 0.24 & I \\
\hline 09 & $\mathrm{CCB}$ & 35 & $\mathrm{~F}$ & SVT++ and RSD & 0.49 & II \\
\hline 10 & JMM & 42 & M & SVT++ & 0.30 & I \\
\hline 11 & MRS & 56 & $\mathrm{~F}$ & SVT +++ & 0.38 & II \\
\hline 12 & JEE & 52 & M & SVT+++ & 0.28 & I \\
\hline 13 & SAA & 46 & $\mathrm{~F}$ & SVT++++ & 0.34 & III \\
\hline 14 & $\mathrm{CCN}$ & 62 & M & SVT+++ & 0.35 & III \\
\hline 15 & $\mathrm{JRB}$ & 67 & M & SVT++++ & 0.21 & III \\
\hline
\end{tabular}


Simultaneously, four leads of the surface electrocardiogram (ECG) (D1, aVF and/or D3, V1 and V5 or V6) were recorded by a multiple channels, as well as the intracavitary electrograms of the following sites: His bundle and, sequentially, HRA and RV in velocities of 25 and $100 \mathrm{~ms}$. The recording systems SP12 (Tecnologia Eletrônica Brasileira$\left.\mathrm{TEB}^{\circledR}\right)$ and LabSystem - version $2.56\left(\right.$ BARD $^{\circledR}$ Electrophysiology) were used, as well as the recordings of bipolar electrograms with filters of 100 to $500 \mathrm{~Hz}$. Bipolar recordings were obtained between the extremity and the second electrode of the quadripolar catheters. When the TEB ${ }^{\circledR}$ polygraph was used, if the stimulation was performed by the pair of distal electrodes, the recording was achieved from the proximal pair of electrodes. These recordings were obtained with variable gains, maintaining a fixed pattern $\mathrm{N}: 10 \mathrm{~mm}=1 \mathrm{mV}$.

The protocol of programmed ventricular stimulation was performed with the double value of the diastolic stimulation threshold, in two basic cycles ( 450 and $600 \mathrm{~ms}$ ) in the apex and RV outflow tract, applying up to three early extrastimuli (Fisiocor Cardiostimulator-Cardiobras Ltda). None of the patients studied needed to receive isoproterenol forSVT induction.

When tachycardia resulted in major hemodynamic impairment, it was immediately reverted by the entrainment technique, employing early extrastimuli or external electric cardioversion. Then, $1.0 \mathrm{~g}$ of procainamide was administered given intravenously for a 20 -minute period aiming to slow the tachycardia and to make it hemodynamically stable in its reinduction.

For endocardial mapping of the LV, puncture of the femoral artery was performed, by which a deflectable 7F quadripolar catheter was introduced, with interelectrode interval of 2-5-2mm and extremity of $8 \mathrm{~mm}$ (Blazer, EP Technologies, Mountain View, CA), placed in the LV by retrograde via through the aortic valve. The position of the catheter was determined by fluoroscopy, using right anterior oblique $\left(30^{\circ}\right)$ and left anterior oblique $\left(30^{\circ}\right.$ to $\left.45^{\circ}\right)$ views. After arterial puncture, heparin was intravenously administered as follows: 5,000 $\mathrm{U}$ in bolus and 1,000 $\mathrm{U}$ per hour during the whole procedure.

To identify the sites of SVT, the mapping scheme described by Josephson was used: site 1 is the apex; sites 2, 3 and 4 are the septum; sites 5 and 6 represent, respectively, the mid and basal inferior wall; site 7, the apical lateral wall; site 8 , the inferoposterior region; site 9 , the apical anterolateral wall; site 10, the basal lateral wall; site 11, the midanterior wall ; site 12, the basal anterior wall; and sites 13 to 18 represent the right ventricle. Segmental areas of 5 to $10 \mathrm{~cm}^{2}$ represent each site ${ }^{14}$.

In all patients, mapping was performed during tachycardia under continuous intraarterial monitoring. The endocardial mapping was initially directed to the detection of fractionated electrical activity with presystolic activity of at least $30 \mathrm{~ms}$ or of mid-diastolic potentials. In order to evaluate the possibility to demonstrate the participation of these potentials in the reentrant circuit by ventricular stimulation during tachycardia, entrainment was performed with the greatest cycle length capable of penetrating the circuit of tachycardia. In these sites, it was usually necessary to use the maximum energy to achieve ventricular depolarization. In the stimulated sites, the presence or absence of fusion of the QRS complexes was analyzed. Measurements of the differences between the values of the return cycle and the cycle length of SVT, and between stimulus to QRS interval and the presystolic activity were performed. Differences up to $20 \mathrm{~ms}$ between these measurements were not considered.

The RF current was released between the distal electrode of the multipolar catheter and a shovel-shaped external electrode placed on the back of the patient's thorax, with the power of 20 to $30 \mathrm{~W}$ during 15 to 60 s, without temperature control. During RF ablation, impedance was continuously measured, being the system automatically powered off if the impedance reached a value $\geq 150 \mathrm{Ohms}$.

In all sites of RF current application, the characteristics of the intracavitary electrograms were analyzed in regard to presystolic activity, amplitude and duration, and they were compared to each other in the sites of successful and unsuccessful RF current application. In stimulated sites of RF current application, where entrainment of the SVT was obtained, we analyzed the presence of concealed entrainment and the difference between the following intervals: a) return cycle and SVT cycle length b) the stimulus to QRS interval and presystolic activity.

For analyzing the results, the following tests were applied: 1) Mann-Whitney's test ${ }^{16}$ with approximation to normal curve in order to compare the successful and unsuccessful groups in regard to the variables studied; 2) Chi-square test in order to associate success and failure with the electrophysiological variables studied. When Cochran constraints were observed, the Fishers exact test was applied ${ }^{16}$.

The level of rejection of the null hypothesis was established to be 0.05 or $5 \%(\alpha \leq 0.05)$, and the significant values were signaled with an asterisk.

\section{Results}

From an initial population of 31 patients, only 15 could be included in the study, all of them coincidentally with recurrent and refractory SVT to at least one class III antiarrhythmic drug. From the remaining 16 patients not included on antiarrhythmic drug therapy, eight had recurrent SVT, and eight had a clinical course without recurrence.

In regard to the patients with recurrent SVT of the group excluded, it was not possible to identify, in three of them, a site of presystolic activity using the endocardial mapping techniques. In the other five patients, there was reproducible induction of a fast SVT with hemodynamic instability. These patients required repetitive electrical cardioversions, which made the mapping of the clinical SVT impossible. All the eight patients without clinical recurrence under drug treatment showed, in the laboratory, a major 
hemodynamic instability during tachycardia similar to the clinical one, and they could not be mapped.

During endocardial mapping of the 15 patients studied, 17 distinct ventricular tachycardia sites were identified. The cycle of the mapped tachycardias varied from $300 \mathrm{~ms}$ to $460 \mathrm{~ms}$ ( $\mathrm{mean}=368 \pm 64 \mathrm{~ms}$ ) and, in three patients, hemodynamic stability during SVT was achieved after endovenous administration of procainamide. The $3^{\text {rd }}$ extrastimulus was necessary to the induction of four out of the 17 tachycardias $(23.5 \%)$.

Radiofrequency current application was performed in sites with presystolic activity varying from $30 \mathrm{~ms}$ to $220 \mathrm{~ms}$. Presystolic activity of ventricular electrograms in the sites of unsuccessful RF ablation was significantly greater than in the sites of success. Regarding the characteristics of the electrograms, all successful RF ablation sites showed continuous electrical activity (fig. 1) or mid-diastolic potentials (fig. 2A) or fractionated electrograms with presystolic activity (fig. 3A). Duration of electrograms in the successful (158ms) and unsuccessful (155ms) sites showed no statistically significant difference. In the same way, the amplitude of the electrograms in successful $(0.40 \mathrm{mV})$ and unsuccessful $(0.51 \mathrm{mV})$ sites showed no significant difference.

Entrainment of all mapped SVTs was achieved, confirming the reentry mechanism. In 14 of the 17 sites ( $82 \%)$, it was possible to show the participation of the fractionated mid-diastolic or presystolic electrical potentials, which were recorded from endocardium with the tachycardia circuit through the following electrophysiological mapping parameters: concealed entrainment with return cycle approximately equal to the SVT cycle-length and stimulus to QRS interval similar to the presystolic activity (fig. 2B, 3B and 4).

Localization of the reentrant circuit was confirmed in 12 sites of SVT $(70.5 \%)$ by its termination at the first $15 \mathrm{~s}$ of RF current application (fig. 5) and in two other sites by transitory slowness of the tachycardia during the release of energy (fig. 6). Most of the sites were located at the inferoposterior dorsal (site 8) and lateral (sites 7 and 10) wall. The characteristics of SVT and of its site of origin are described in table II.

Entrainment was performed at 64 of the 174 sites of RF application. A significant association between concealed entrainment and the success of RF ablation was observed, with $53.5 \%$ of success of the 28 sites where this parameter was obtained versus $5.5 \%$ of success of the 36 sites of entrainment with fusion (tab. III). A significant association of the return cycle length to the tachycardia cycle with the success of RF ablation was also observed, with $35.7 \%$ of success of 42 sites where this parameter was obtained versus $9.0 \%$ of success of the 22 sites where the return cycle was more than $20 \mathrm{~ms}$ greater than the tachycardia cycle length (tab. IV). However, in the sites where concealed entrainment was achieved, the rate of success of the sites with return cycle similar to the SVT cycle was not significantly different from that at the sites with a return cycle more than $20 \mathrm{~ms}$ greater than the tachycardia cycle (53.8\% versus $50.0 \%$ ). This rate was similar to that achieved when the overall rate of success of the sites was analyzed with concealed entrainment $(53.5 \%)$, i.e., regardless of the analysis of the return cycle (tab. V). No association between stimulus to QRS complex interval similar to the presystolic activity and the success of the RF ablation was observed either isolated or associated with concealed entrainment achievement.

From the three tachycardias in which it was not possible to reverse or change the cycle during endocardial RF application, two had left bundle-branch block (LBB) morphology (66.6\%). On the other hand, from the 14 SVTs with right bundle-branch block (RBB) morphology (7.2\%), it was not possible in only one to reach the circuit through RF. There was a nonsignificant trend in tachycardia circuits with LBB morphology to be less accessible to the lesion produced by endocardial RF current application than those with RBB morphology.

\section{Discussion}

Population studied - In order to map an SVT, it needs to be hemodynamically stable. In this study, only the patients with recurrent SVT using antiarrhythmic drugs

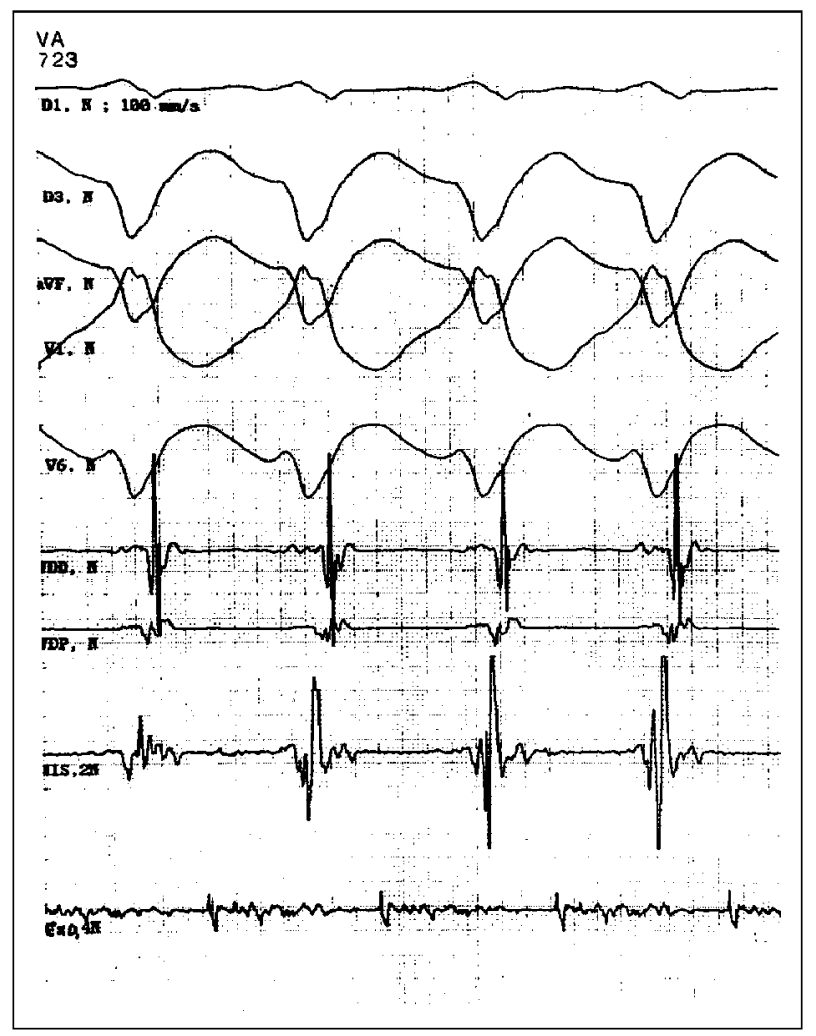

Fig. 1 - Endocardial mapping during ventricular tachycardia (VT). Intracavitary record of the right ventricle by distal and proximal electrode pairs (VDD and VDP, respectively), of the His bundle (HIS) and of the left ventricle by distal electrode pair of the explorer catheter (EXD). In this one, record of continuous electrical activity is obtained. RF blation at this site resulted in immediate VT termination. $\mathrm{N}: 10 \mathrm{~mm}=1 \mathrm{Mv}$. 


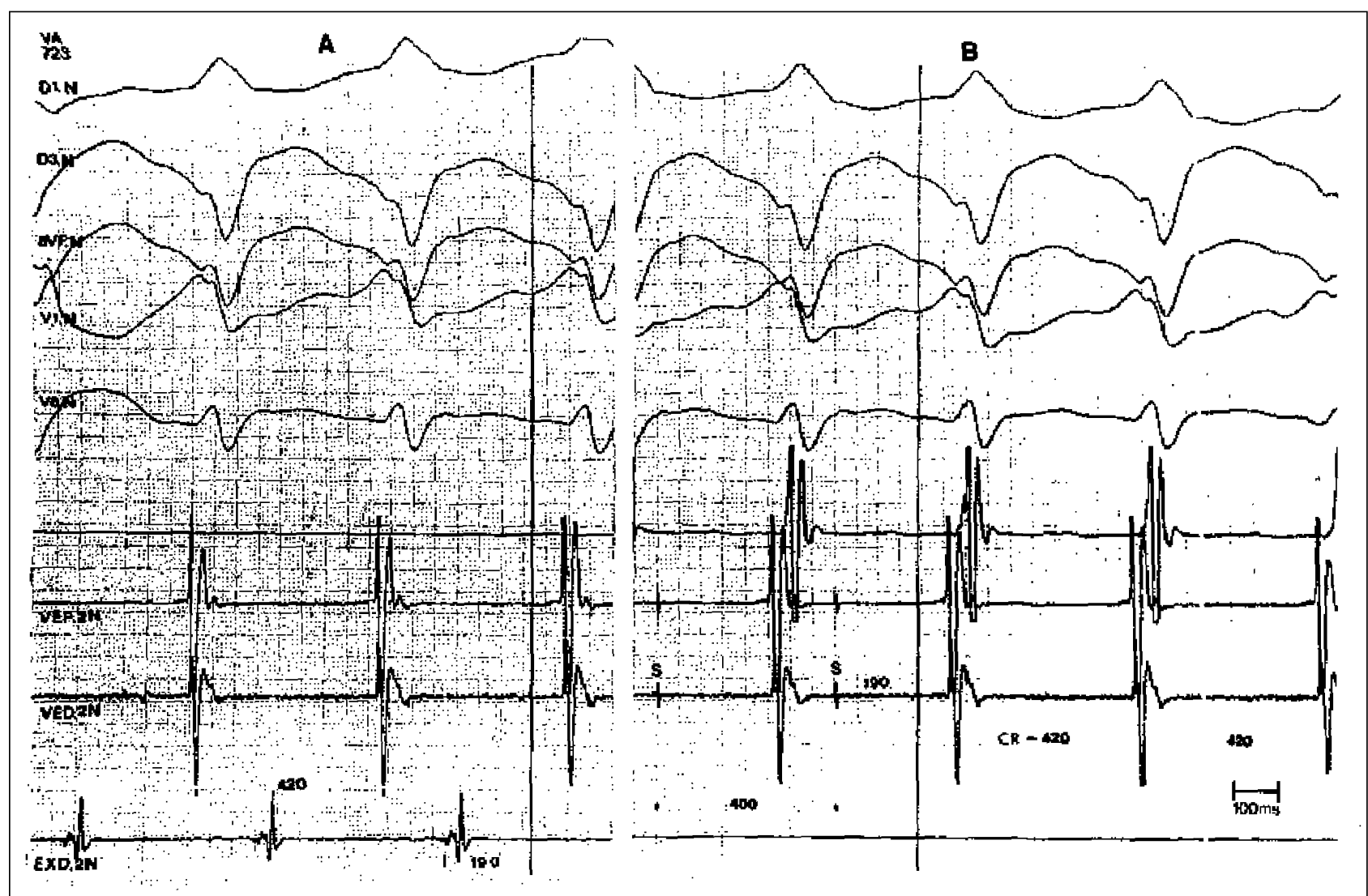

Fig. 2 - Endocardial mapping during ventricular tachycardia (VT), using the entrainment technique. Record of surface leads D1, D3, aVF, V1 and V6, and right and left ventricle intracavitary electrograms by two catheters: one through the distal electrode pair (EXD) and another through the proximal and distal electrode pairs (VEP and VED, respectively) The traces mark the QRS complex onset. Panel A: through catheter EXD, a mid-diastolic potentials recorded with 190ms of presystolic activity regarding the QRS complex onset. Panel B: stimulation through the EXD catheter with a 400ms cycle length (20 ms less than that of the VT) produces concealed entrainment, return cycle equal to VT cycle length and stimulus (S) to QRS interval equal to the mid-diastolic potential presystolic activity, confirming its participation in the reentrant circuit. $\mathrm{N}: 10 \mathrm{~mm}=1 \mathrm{mV}$.

were mapped. In the group without clinical recurrence under medicamentous therapeutics, it was only possible to induct, by programmed ventricular stimulation, fast tachycardia with major hemodynamic repercussion.

It is well known that, for a ventricular tachycardia to manifest itself in a sustained manner, it is necessary that the conduction time of the activation wave through the circuit be sufficiently long to allow it, when leaving the circuit, to reach the ventricle out of its refractory period. Therefore, increasing the ventricle refractory period in patients with fast circuits, we have a greater chance of preventing the activation wave, when leaving the circuit, from depolarizing the ventricle and triggering an SVT. Unfortunately, drugs increase the refractory period at most $50 \mathrm{~ms}{ }^{17}$. Therefore, in many patients with fast SVT, the effect of these drugs on the excitable gap of the circuit and on the ventricular refractory period is not enough to prevent an activation wave from triggering a ventricular tachycardia by reentry.

Josephson ${ }^{17}$ observed in his laboratory that class I drugs were more effective in preventing fast SVT reinduction (cycle $\leq 270 \mathrm{~ms}$ ). Marchlinski et al ${ }^{18}$ showed that the effects of amiodarone and procainamide on the SVT cycle are similar, suggesting the possibility of using the response to programmed ventricular stimulation with procainamide to predict the clinical results of amiodarone. The same technique was used by Winters et al ${ }^{19}$ in an attempt to predict the clinical effectiveness of sotalol. In their study, SVTs that became non-inducible with sotalol responded in two ways to procainamide: either they were not inducible or the cycle of tachycardia increased significantly. In the latter, the average of the SVT cycle length was smaller $(246 \pm 35 \mathrm{~ms})$ than in the nonresponsive ones to both drugs $(294 \pm 58 \mathrm{~ms})$. In our service, among chagasic patients with spontaneous SVT, clinical control was observed to be more often significantly achieved in patients with SVT with a cycle $\leq 270 \mathrm{~ms}$ than in those whose cycle was $>270 \mathrm{~ms}$, during programmed ventricular stimulation in basal conditions ( $\mathrm{p}=0.003$ ), after a follow-up period of $36 \pm 27$ months using amiodarone or sotalol ${ }^{20}$. These data suggest that the tachycardia cycle can be a decisive factor to medicamentous effectiveness.

For these reasons, it is expected that recurrent SVTs, which are refractory to drugs that act predominantly prolonging the refractoriness, are slower (cycle $>270 \mathrm{~ms}$ ), providing a greater chance of hemodynamic tolerance and making the mapping possible. This fact might explain why, of 


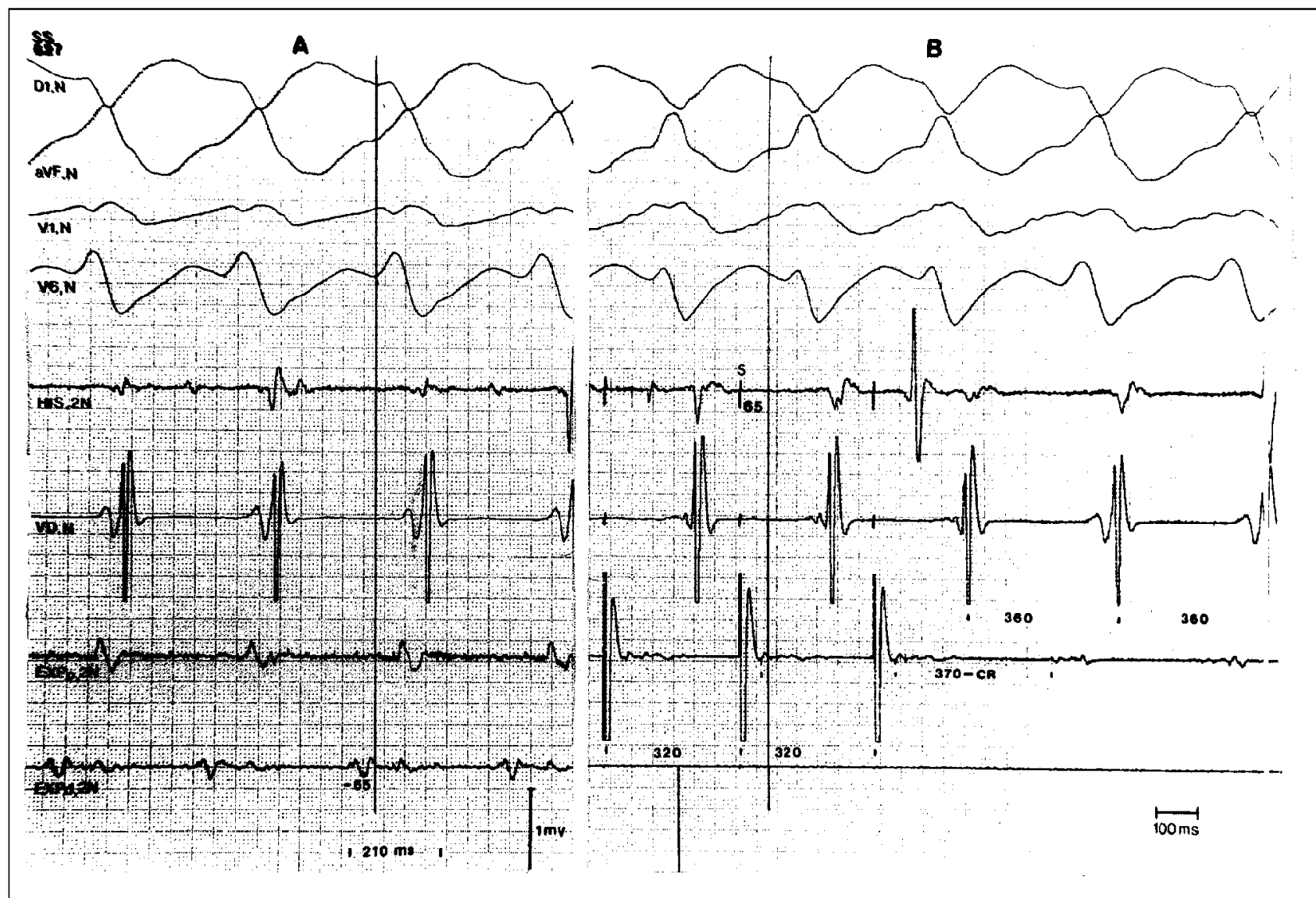

Fig. 3 - Endocardial mapping during ventricular tachycardia (VT) using the entrainment technique. Record of surface leads D1, aVF, V1 and V6, and of the intracavitary electrograms of the His bundle (HIS), of the right (VD) and left ventricles through the proximal and distal electrode pairs (EXP and EXD, respectively). The traces mark the QRS complex onset. Panel A: by EXD catheter, the fractionated electrical activity record (multiple components, $210 \mathrm{~ms}$ extension and amplitude $<0.5 \mathrm{mV}$ ) is obtained. Panel B: stimulation through catheter EXD with a $320 \mathrm{~ms}$ cycle (40ms less than that of the VT) produces concealed entrainment, return cycle (CR) similar to the VT cycle length and the stimulus (S) to QRS interval equal to the presystolic activity, confirming its participation in the reentrant circuit. $\mathrm{N}: 10 \mathrm{~mm}=1 \mathrm{mV}$.

the eligible patients in this study, only those with recurring SVT under antiarrhythmic therapeutics could be mapped.

Ventricular function and heart rate during SVT are the variables that influence most hemodynamic tolerance to tachycardia. EF evaluated by Doppler echocardiogram of the patients with tolerated SVT was similar to that from those resulting in severe hemodynamic impairment. However, the cycle length of inducible SVT in patients resulting in severe hemodynamic impairment varied from 250 to $340 \mathrm{~ms}($ mean $=280 \pm 31.3 \mathrm{~ms})$ and that of hemodynamically tolerated SVTs varied from 320 to $400 \mathrm{~ms}$ (mean = $325.5 \pm 27 \mathrm{~ms}$ ), suggesting that the cycle length of SVTs is the most important factor for hemodynamic stability during tachycardia.

Programmed ventricular stimulation - Since its introduction into clinical practice, the electrophysiological study has contributed to a better understanding of ventricular arrhythmias, allowing great advances in pharmacological and non pharmacological therapeutics. In the present study, the ventricular stimulation protocol with three extrastimuli was used. Employment of the $3{ }^{\text {rd }}$ stimulus has been necessary to reproduce clinical SVT in up to $42 \%$ of the chagasic patients, mainly when the SVT cycle is $\leq 280 \mathrm{~ms}^{21}$. Among patients included in this study, there was less of a need to use the $3^{\text {rd }}$ extrastimulus to reproduce clinical SVT (23\%). Therefore, despite the use of drugs prolonging the refractoriness, the excitable gap of the circuits of the patients studied seems to remain wide. In this study, the $3^{\text {rd }}$ extrastimulus was also greatly used in the postablation phase, allowing a more reliable evaluation of the response to the procedure.

Endocardial mapping-Mapping techniques during sinus rhythm were not used in the patients studied because they proved to be unsatisfactory for the detection of SVT circuits. Abnormal electrograms in sinus rhythm have high sensitivity $(86 \%)$ but low specificity $(65 \%)$ in detecting SVT circuits. The specific electrograms (fractionated and late) have good specificity (90\% and $95 \%$, respectively) but low sensitivity (10\% and $20 \%$, respectively) ${ }^{13}$. The technique of ventricular stimulation in sinus rhythm has many distortions: 1) ventricular stimulation in adjacent sites ( 2 to $3 \mathrm{~cm}$ ) may produce the same morphology of clinical SVT or roughly different configurations, mainly regarding the electrical axis change; 2) stimulation of the same site in different times may 


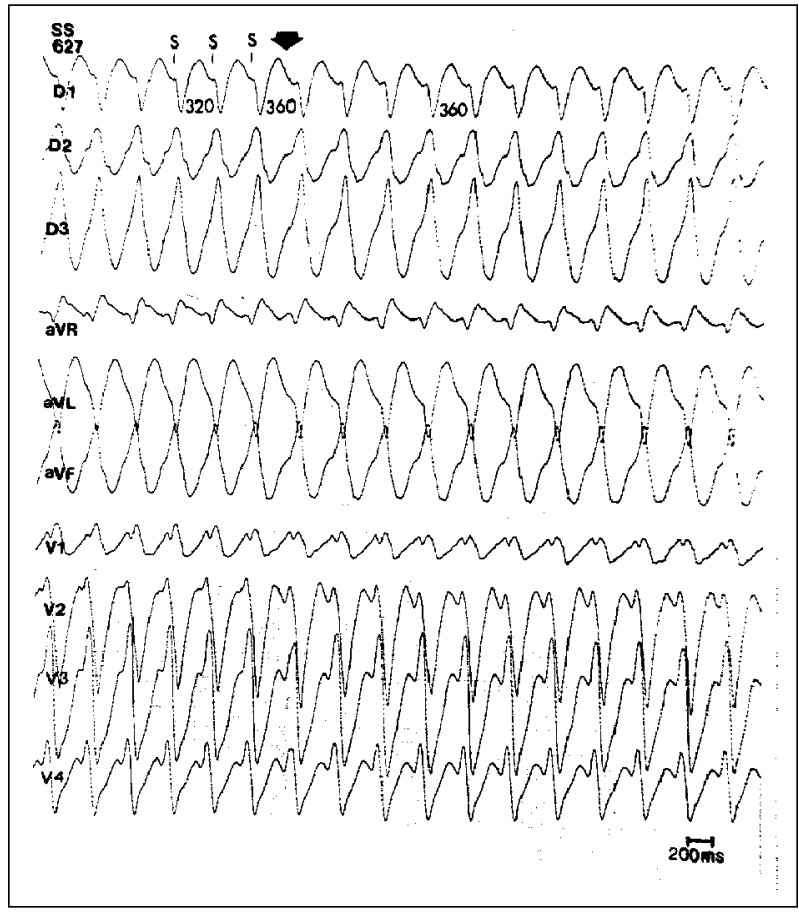

Fig. 4 - Surface electrocardiogram at $25 \mathrm{~mm} / \mathrm{s}$ obtained during stimulation (S) of the endocardial site recorded by the EXD catheter of figure $3 \mathrm{~A}$ and when stimulation was stopped (from the arrow). It is observed that stimulation produces concealed entrainment without changing VT morphology in any recorded lead, and return cycle (arrow) equal to that of VT. $\mathrm{N}: 10 \mathrm{~mm}=1 \mathrm{mV}$.

generate distinct morphologies; for instance, the stimulation of the septum at the same site may occasion RBB and LBB morphology, depending on the preferential transeptal conduction to LV or RV, respectively ${ }^{22-24}$.
The most reliable and used mapping method has been the determination in multiple places of the sequence of ventricular activation during tachycardia. Therefore, SVT mapping of the patients studied was performed during tachycardia and directed to detect the slow conduction zone of the reentrant circuit, as described below. In our study, entrainment of all SVT mapped was obtained. This method, in addition to confirming the reentrant mechanism, shows the involvement of presystolic fractionated electrical potentials with the slow conduction zone of the circuits.

Intracavitary electrograms - In our patients, the presence of fragmentation was considered important. According to Josephson et al ${ }^{20}$, during tachycardia, low amplitude electrograms are invariably observed, with multiple components and increased duration in the endocardial sites of SVT. These electrograms represent the activation of a slow conduction zone. Due to specific electrophysiological characteristics, it is the only part of the circuit that may be identified by means of endocavitary electrodes. When participating in the circuit, fractionated electrograms are always related to $\mathrm{QRS}$ complex, and may be found bound to it (presystolic activity) or separated from it by an isoelectrical line (mid-diastolic potentials) ${ }^{13}$. The earliest electrical activity may be recorded along a relatively large area, distal to the outlet site of the reentrant circuit, sometimes concomitantly in the endocardium and epicardium. This suggests that the detection of presystolic electrical activity is not enough for circuit identification, as experimentally shown by El Sherif et $\mathrm{al}^{25}$ in animal models. In this study, the activation wave leaving the reentrant circuit depolarized adjacent regions not necessary for

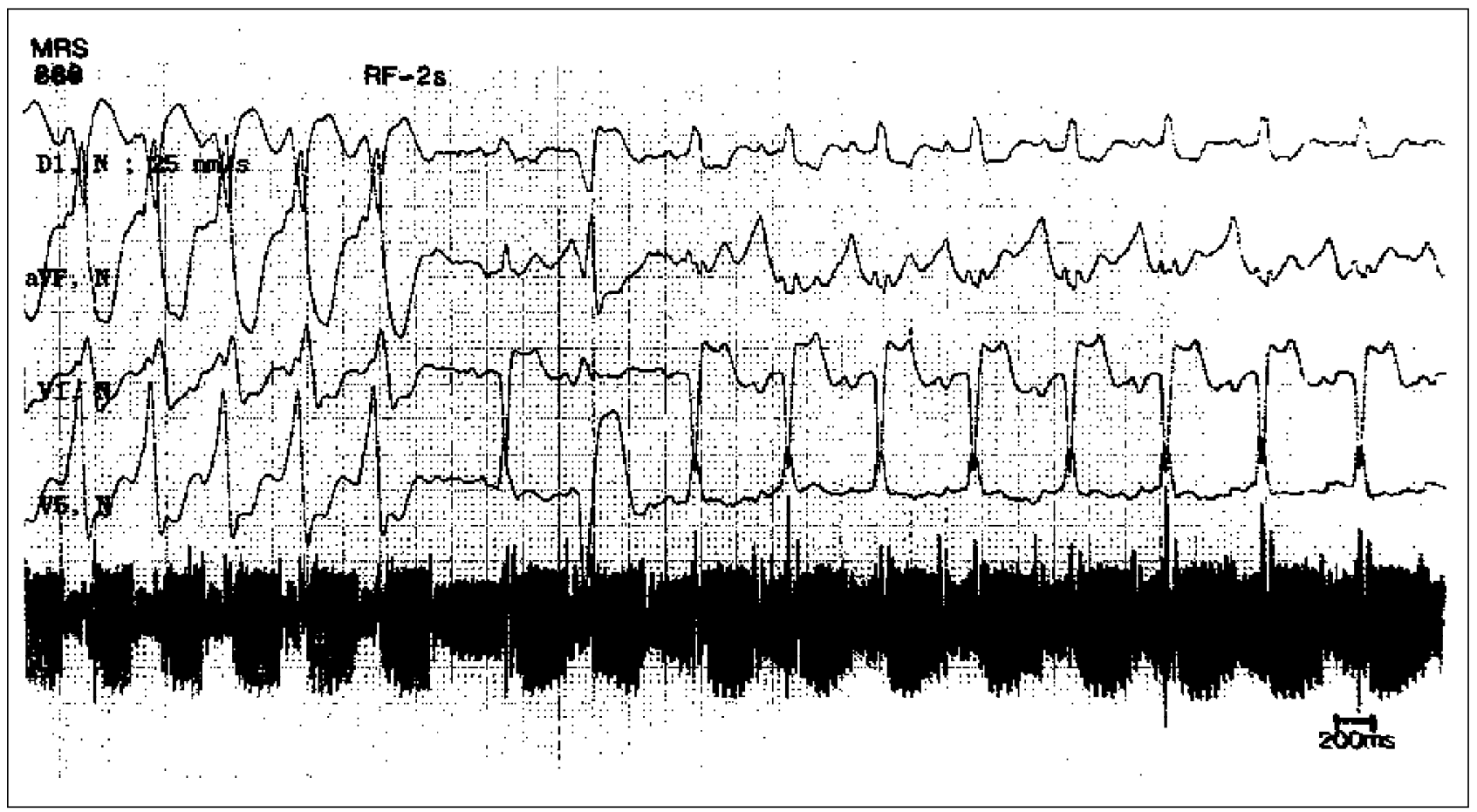

Fig. 5 -Termination of ventricular tachycardia is observed in the first $2 \mathrm{~s}$ of the onset of radiofrequency current application. 


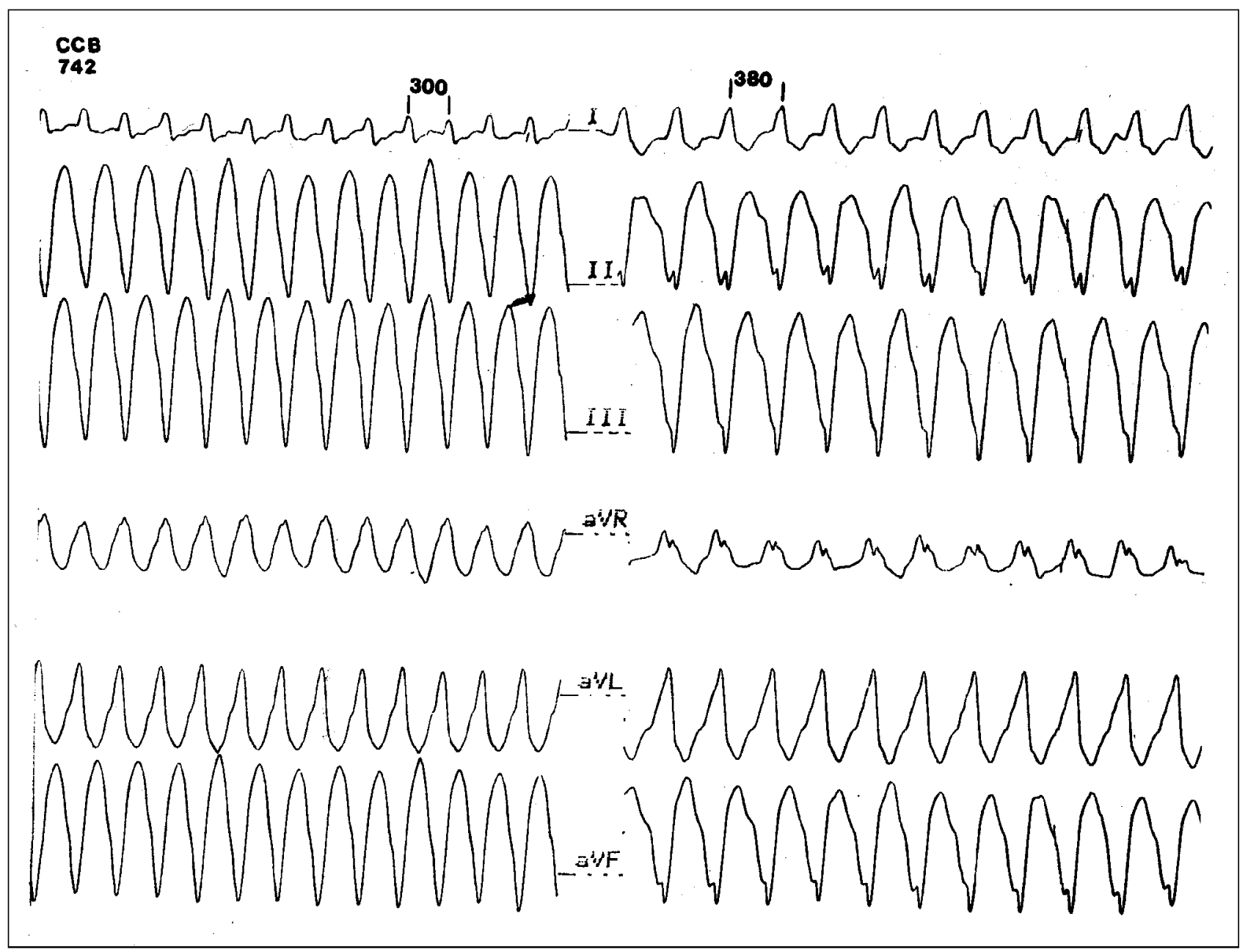

Fig. 6 - During radiofrequency application, the tachycardia cycle length is made slower, from $300 \mathrm{~ms}$ to $380 \mathrm{~ms}$.

\begin{tabular}{|c|c|c|c|c|c|c|c|c|}
\hline \multirow[t]{2}{*}{ Pts } & \multicolumn{3}{|c|}{ SVT } & \multirow[t]{2}{*}{ Site } & \multirow[t]{2}{*}{$\mathrm{RF}$} & \multicolumn{3}{|c|}{ Entrainment } \\
\hline & Morph & Axis & $\mathrm{CL}(\mathrm{ms})$ & & & $\mathrm{CE}$ & $\mathrm{SQRS}=\mathrm{PSA}$ & $\mathrm{RC}=\mathrm{VTCL}$ \\
\hline 1 & $\mathrm{RBB}$ & $120^{\circ}$ & 310 & 8 & S & + & + & + \\
\hline 2 & $\mathrm{RBB}$ & $120^{\circ}$ & 340 & 8 & S & + & + & + \\
\hline 3.1 & $\mathrm{RBB}$ & $120^{\circ}$ & 340 & 10 & $\mathrm{~L}$ & + & + & + \\
\hline 3.2 & LBB & $60^{\circ}$ & 320 & 3 & $\mathrm{~S}$ & + & + & + \\
\hline $4.1^{*}$ & LBB & $120^{\circ}$ & 470 & 12 & I & + & + & + \\
\hline 4.2 & $\mathrm{RBB}$ & $-45^{\circ}$ & 400 & 8 & I & - & + & + \\
\hline 5 & LBB & $-45^{\circ}$ & 320 & 3 & I & - & - & - \\
\hline $6 \#$ & $\mathrm{RBB}$ & $120^{\circ} /-90^{\circ}$ & 460 & 7 & S & + & + & + \\
\hline 7 & $\mathrm{RBB}$ & $120^{\circ}$ & 300 & 11 & $S$ & + & + & + \\
\hline 8 & $\mathrm{RBB}$ & $15^{\circ}$ & 300 & 8 & S & + & + & + \\
\hline 9 & RBB & $-60^{\circ}$ & 300 & 3 & $\mathrm{~L}$ & + & + & + \\
\hline 10 & $\mathrm{RBB}$ & $-45^{\circ}$ & 300 & 7 & $\mathrm{~S}$ & + & + & + \\
\hline 11 & $\mathrm{RBB}$ & $150^{\circ}$ & 380 & 7 & S & + & + & + \\
\hline 12 & $\mathrm{RBB}$ & $120^{\circ}$ & 400 & 8 & $S$ & + & + & + \\
\hline 13 & RBB & $150^{\circ}$ & 430 & 6 & $S$ & + & + & + \\
\hline 14 & RBB & $120^{\circ}$ & 430 & 10 & $S$ & + & + & + \\
\hline $15^{*}$ & RBB & $120^{\circ}$ & 460 & 10 & $S$ & - & - & - \\
\hline
\end{tabular}




\begin{tabular}{|lcccc|}
\hline \multicolumn{5}{|c|}{$\begin{array}{c}\text { Table III - Successful and unsuccessful sites of radiofrequency } \\
\text { current application according to concealed entrainment achievement }\end{array}$} \\
\hline Group & Success & Failure & Total & $\%$ Success \\
\hline Concealed entrainment & 15 & 13 & 28 & 53.5 \\
Entrainment with fusion & 02 & 34 & 36 & 5.5 \\
Total & 17 & 47 & 64 & 26.5 \\
\hline Chi-square test: $\chi^{2}$ calculated $=18.61 * ;$ critical $\chi^{2}=3.84$. \\
\hline
\end{tabular}

tachycardia maintenance, so that cryoablation of these sites generally failed to interrupt the tachycardia.

Later, El-Sherif et al ${ }^{26}$ showed that SVT could be consistently interrupted after slowness and blockade of the conduction, when cryoablation was performed in the distal part of the common via of the circuit proximal to the site of the earliest activity. This showed the need to identify an ideal site in the reentrant circuit in order to achieve effectiveness of the ablation procedure.

In our sample, a value of presystolic activity of at least $30 \mathrm{~ms}$ was required because the activation wave, leaving the outlet site of the circuit, generally crosses a fibrosis region before initiating ventricular depolarization ${ }^{9}$. However, identification of the site of the earliest electrical activity during tachycardia was not useful in precisely identifying the place of its origin; contrarily, significantly earlier presystolic electrical activity was detected in the unsuccessful RF ablation sites than in the successful ones. These data coincide with those of Stevenson et $\mathrm{al}^{9}$, who achieved 33\% success in sites where the stimulus to QRS interval was less than $70 \%$ of the tachycardia cycle length versus $11 \%$, when this interval was greater. According to Josephson et al ${ }^{14}, \mathrm{RF}$ success depends not only on the identification of the slow conduction zone but also on localization of an isthmus in the reentrant circuit, probably more often found near the circuit outlet site. Our findings of greater RF success rates in the regions of smaller presystolic activity are in accordance with these observations.

Amplitude and duration of the electrogram at the sites of ablation were not useful for circuit identification. Fractionated electrograms, despite being essential to circuit identification, were found both in successful and unsuccessful sites of RF application. These data emphasize the need to employ ventricular stimulation techniques to confirm the participation of presystolic fractionated electrograms in ventricular reentry.

\begin{tabular}{|c|c|c|c|c|}
\hline \multicolumn{5}{|c|}{$\begin{array}{l}\text { Table IV - Successful and unsuccessful sites of radiofrequency } \\
\text { current application according to the achievement of the return } \\
\text { cycle (RC) almost equal to the ventricular tachycardia cycle length } \\
\text { (VTCL) in all sites where entrainment was obtained }\end{array}$} \\
\hline Group & Success & Failure & Total & $\%$ Success \\
\hline $\mathrm{RC}=\mathrm{VTCL}$ & 15 & 27 & 42 & 35.7 \\
\hline $\mathrm{RC}>\mathrm{VTCL}$ & 02 & 20 & 22 & 9.0 \\
\hline Total & 17 & 47 & 64 & 26.5 \\
\hline
\end{tabular}

Concealed entrainment - Slow conduction areas of SVT circuits may be clinically evidenced in sinus rhythm and during tachycardia, using ventricular stimulation techniques. Both in sinus rhythm and during tachycardia, stimulation the site of origin, when performed in a slow conduction zone, has a long interval between the stimulus and the local electric activity or the QRS complex ${ }^{27-29}$.

Using the ventricular stimulation technique through concealed entrainment, several authors showed, in patients with chronic ischemic heart disease, the participation of fractionated presystolic activity and mid-diastolic potential records with the slow circuit conduction region ${ }^{6-9,30-33}$. This method was also used to identify the circuit of patients with idiopathic dilated cardiomyopathy ${ }^{33}$.

Intraoperative mappings and computer simulations of endocardial mappings of patients with ischemic heart disease showed that concealed entrainment could be obtained through stimulation of a region of the reentrant circuit. This results in antidromic blockade of the depolarizing wave by the wave front of the tachycardia, preventing depolarization of adjacent regions and also causes the orthodromic conduction of the depolarizing wave in a similar manner to SVT activation ${ }^{34,35}$. In rare situations, blockade of the stimulation wave may occur orthodromically, allowing antidromic conduction and, consequently, change of the outlet site of the circuit and of SVT morphology. This finding can explain the rare situations in which termination of the tachycardia may be obtained during RF application in sites where fusion during SVT entrainment with fusion ${ }^{9}$ is observed. As identification of a endocardial critical region of ventricular reentrant circuits may bring new perspectives to treat this arrhythmia, we used this mapping method in our study, trying to achieve the precise localization of SVT circuits in patients with chronic Chagas' heart disease (CCHD) .

In the 15 patients included in this study, the presence of concealed entrainment through the programmed ventricular stimulation technique was useful in identifying 14 out of 17 reentrant circuits (82\%). Thus, it was shown that, in most of the SVT sites of chagasic etiology, it is possible to perform endocardial stimulation of a zone connected to the reentrant circuit. In this situation, depolarization generated by stimulation depolarizes the ventricle from the same outlet site of the activation wave, so that SVT morphology remains unaltered. This shows that the regions adjacent to

\begin{tabular}{|c|c|c|c|c|}
\hline \multicolumn{5}{|c|}{$\begin{array}{l}\text { Table } V \text { - Successful and unsuccessful sites of radiofrequency } \\
\text { current application according to the achievement of the return } \\
\text { cycle (RC) almost equal to the ventricular tachycardia cycle length } \\
\text { (VTCL) only in sites where concealed entrainment was obtained }\end{array}$} \\
\hline Group & Success & Failure & Total & $\%$ Success \\
\hline $\mathrm{RC}=\mathrm{VTCL}$ & 14 & 12 & 26 & 53.8 \\
\hline $\mathrm{RC}>\mathrm{VTCL}$ & 01 & 01 & 02 & 50.0 \\
\hline Total & 15 & 13 & 28 & 53.5 \\
\hline
\end{tabular}


the common pathway of the circuit are not depolarized. Therefore, the stimulated area is protected by anatomic or functional barriers that prevent the radial diffusion of the stimulus.

In patients with coronary artery disease, Morady et al ${ }^{36}$ using only concealed entrainment as a criterion for RF ablation, obtained success in only $56 \%$ of cases. Stevenson et $\mathrm{al}^{9}$ reported that, in concealed entrainment sites, success in terminating tachycardia was obtained in $17 \%$ of cases. In the same study, the presence of the following associated parameters predicted the success of RF application in $35 \%$ of the sites: mid-diastolic potentials, continuous electrical activity, concealed entrainment with return cycle nearly equal to the SVT cycle length, the stimulus to QRS interval $>60 \mathrm{~ms}$ and $<70 \%$ of the SVT cycle length. When none of these criteria were found, sucess of RF application was $4 \%$.

In the present study, RF application in places of fractionated presystolic activity has resulted in SVT termination in $29.3 \%$ of the sites. In the places where concealed entrainment was achieved, it was possible to terminate tachycardia during RF application in $53.5 \%$ of the sites versus $5.5 \%$, when this criterion was not obtained. Concealed entrainment detection was the best parameter for identifying the tachycardia circuit.

Return cycle versus sustained ventricular tachycardia cycle length - In computer simulations of endocardial mapping, Stevenson et $\mathrm{al}^{9}$ found that, in addition to stimulation in the common pathway of the circuit, the presence of concealed entrainment could occur in regions bound to the circuit, but not participating in it, named bystander areas, where RF application is not effective in terminating SVT. The authors observed that, when stimulation was performed in the common via, in addition to the similarity between spontaneous heart beat morphology and that of the stimulated heart beat, the return cycle is nearly equal to the SVT cycle. The return cycle represents the conduction time from the stimulated site to the circuit and the return to the same site. Therefore, when stimulation is performed out of the reentrant circuit, the return cycle is greater than that of the SVT. However, this situation may also occur when the stimulation is performed in the common pathway of the circuit, due to the decremental properties of the slow conduction zone of the reentry.

In order to avoid conduction delay due to faster stimulation of the common circuit via, mimicking a bystander area, the entrainment was performed in the greatest cycle able to entrain the circuit. Due to decremental properties, increments of up to $20 \mathrm{~ms}$ in the return cycle in relation to that of the SVT cycle length and the stimulus to QRS interval regarding the presystolic activity were considered insignificant. In patients with coronary artery disease, a return cycle of up to $30 \mathrm{~ms}$ greater than the SVT cycle, under concealed entrainment, was a strong predictor of SVT termination by RF energy ${ }^{9}$.

In the present study, termination of SVT by RF was also associated with achievement of the return cycle similar to that of the SVT, regardless of the presence of concealed entrainment, but with a lower success rate $(35.7 \%)$ than that achieved by analysis of concealed entrainment (53.5\%). However, analysis of the return cycle in sites with concealed entrainment did not show any usefulness for circuit identification, and the same success rates obtained when only the concealed entrainment parameter was considered were observed, suggesting that the circuits of chagasic etiology have few bystander areas. Thus, the analysis of the return cycle in these sites is useful only when discrete alterations of amplitude and duration of the QRS complexes during ventricular stimulation are observed, which may occur due to anisotropic ventricular conduction, giving a false impression of fusion. In this situation, the analysis of the return cycle may be an indicator that stimulation is being performed in the reentrant circuit, even in the presence of a discrete alteration of the QRS complex during stimulation. In patients with coronary artery disease, Stevenson et al ${ }^{9}$ achieved a greater success rate when they associated the analysis of the return cycle with the detection of concealed entrainment (25\% versus $17 \%$, respectively), showing that bystander areas are more often found in these patients than in the chagasic ones.

Stimulus to QRS interval versus presystolic activity - Stimulation of presystolic activity sites related to the reentrant circuit may produce an interval between the stimulus and the QRS complex similar to the presystolic activity and greater than this one, when performed in bystander areas ${ }^{9}$. However, these authors found that, in $49 \%$ of the sites related to the reentrant circuit where there was concealed entrainment with return cycle similar to the SVT cycle length and successful RF application, the identity between presystolic activity and the stimulus to QRS interval was not present. They suggested, then, that this discrepancy could occur due to discrete alterations of the pericircuit conduction, localized in the region between the circuit outlet site and the outlet of the scar region, in an insufficient degree to influence SVT morphology. It was, then, postulated that a fan-shaped configuration at this place explains these discrete changes of velocity of conduction of the activation wave through the scar region.

In the present study, the stimulus to QRS interval onset almost equal to the presystolic activity was not associated with successful RF application. Therefore, this analysis was not useful in helping to identify the circuit of tachycardias. The difficulty in precisely identifying the QRS complex onset during stimulation and the delay of the stimulus conduction in the region between the circuit outlet and the onset of ventricular depolarization (scar) could justify this finding.

Bystander areas - In the study by Stevenson et al ${ }^{9}$, the analysis of the return cycle and the stimulus to QRS interval suggested that $25 \%$ of the sites with concealed entrainment were bystander areas. In our patients, using the same criteria, only 2 out of the $28(7 \%)$ sites with 
concealed entrainment suggested bystander areas. This fact may be due to the specific characteristics of the region of fibrosis of the arrhythmogenic substrate with chagasic etiology. In patients with coronary artery disease, the scar region generally constitutes a compact area, from where several viable myocardial cell branches separated by fibrous tissue may arise, originating the reentrant circuits. In chagasic patients, however, fibrotic regions originated from independent multifocal inflammatory processes are not involved in a compact fibrotic zone. This fibrotic zone in patients with coronary artery disease would favor the isolation of fibrotic areas connected to the circuit, but not pertaining to it, from the remaining myocardium, forming the circuit bystander regions. Lack of isolation of these fibrotic areas in chagasic patients, may result in radial diffusion of the activation wave during stimulation with fusion of the QRS complexes. It seems that, in circuits of chagasic etiology, only the common circuit via is separated from the viable adjacent myocardium, whose stimulation produces entrainment with concealed fusion.

The presence of slow conduction vias comprising large areas of surviving myocardium, as shown by Downar et $\mathrm{al}^{37}$ in patients with coronary artery disease, can justify the unsuccessful ablation in sites with concealed entrainment. This evidence emphasizes the importance of recognizing an isthmus in the reentrant circuit, where the lesion produced by RF causes conduction blockade. New methods making possible a homogeneous lesion of greater extent will increase the success rates of this clinical entity.

Morphology of the sustained ventricular tachycardia versus endocardial identification of the circuit - Endocardial identification of the reentrant circuit through RF ablation was not obtained in three sites. From these, two had LBB morphology. The sites of SVT with this morphologic pattern are usually located in the septum with prefe- rential conduction to RV. Thus, they may be located more in the intramural space than in the endocardial one, which justifies the fact that these tachycardias were less accessible to the lesion produced by endocardial RF current.

Sites of origin of SVT - In our patients, as well as those discussed previously in other studies of this disease ${ }^{38,39}$, the most frequent locations of the sites of SVT origin were the inferoposterior and lateral regions. Even though the sites of SVT origin may be identified by detection of presystolic activity, only electrophysiological parameters obtained by the entrainment technique make possible the precise identification of SVT reentrant circuit ${ }^{9,14}$. The posterior region is one of the most compromised, probably because it is one of the first to undergo inflammation in chagasic heart disease ${ }^{2}$.

Conclusions - In the sample studied, the reentrant mechanism was responsible for SVT genesis in all patients. In $70.5 \%$ of the sites of SVT studied, the endocardial participation of the slow conduction zone of the ventricular reentrant circuit was shown by achieving concealed entrainment with a return cycle similar to that of SVT cycle length and tachycardia termination in the first $15 \mathrm{~s}$ of RF current application.

Successful sites of RF current application showed presystolic activity significantly less than that at unsuccessful sites, showing that the isthmus of the circuit is closer to its outlet site. Concealed entrainment achievement was the main electrophysiological parameter associated with success of RF ablation to terminate SVT. Analysis of the return cycle and of the stimulus to QRS interval during concealed entrainment was not necessary to identify the reentrant circuit; therefore, electrophysiological evidence of bystander areas was not found.

\section{References}

1. Comité de Expertos de La Oms En El Control de La Enfermedad de Chagas Distribución geográfica y prevalencia de la infección en el ser humano. in comité de expertos de la OMS en el control de la enfermedad de Chagas: informe de un Comité de Expertos de la OMS - Control de la enfermedad de Chagas. Série de informes técnicos de la OMS, Spain 1991; 811: 29-32.

2. Anselmi A, Moleiro F, Mendonza I - Cuadro clínico de la enfermidad miocárdica de Chagas. Diagnóstico diferencial con la miocardiopatía dilatada o congestiva. Rev Lat Cardiol 1982; 3: 97-104.

3. Lopes ER, Chapadeiro E - Morte súbita em área endêmica da doença de Chagas. Rev Soc Bras Med Tropical 1983; 16: 79-84.

4. Mujica IJM - Muerte subita y enfermedad de Chagas. Rev Fed Arg Cardiol 1988; 17: 222-3.

5. Gonska B-D, Brune S, Bethge KP, Kreuzer H - Radiofrequency catheter ablation in recurrent ventricular tachycardia. Eur Heart J 1991; 12: 1257-65.

6. Gonska B-D, Cao K, Schaumann A, Dorszewski A, Von Zur MF, Kreuzer H Catheter ablation of ventricular tachycardia in 136 patients with coronary artery disease: results and long-term follow-up. J Am Coll Cardiol 1994; 24: 1506-14.

7. Morady F, Harvey M, Kalbfleisch SJ, El-Atassi R, Calkins H, Langberg JJ Radiofrequency catheter ablation ventricular tachycardia in patients with coronary artery disease. Circulation 1993; 87: 363-72.
8. Gürsoy S, Chiladakis I, Kuck K-H - First lessons from radiofrequency catheter ablation in patients with ventricular tachycardia. PACE 1993; 16: 687-91.

9. Stevenson WG, Khan H, Sager P, et al - Identification of reentry circuits sites during catheter mapping and radiofrequency ablation of ventricular tachycardia late after myocardial infarction. Circulation 1993; 88: 1647-70.

10. Borggrefe M, Chen X, Hindricks G, et al - Catheter ablation of ventricular tachycardia in patients with heart disease. In: Zipes DP - Catheter Ablation of Arrhythmias. Armonk, New York: Futura Publishing, 1994: 277-308.

11. Chen X, Borggrefe M, Hindricks G, et al - Radiofrequency catheter ablation of recurrent ventricular tachycardia related to coronary heart disease: acute, early and long-term efficacy. Circulation 1994; 90: I-558.

12. Kim YH, Sosa-Suarez G, Trouton TG, et al - Treatment of ventricular tachycardia by transcatheter radiofrequency ablation in patients with ischemic heart disease. Circulation 1994; 89: 1094-102.

13. Cassidy DM, Vassalo JA, Buxton AE, Doherty JU, Marchlinski FE, Josephson ME - The value of catheter mapping during sinus rhythm to localize site of origin of ventricular tachycardia. Circulation 1984, 69: 1103-10.

14. Josephson ME - Recurrent ventricular tachycardia. In: Josephson ME - Clinical Cardiac Electrophysiology: Techniques and Interpretations. $2^{\text {nd }}$ ed. Philadelphia: Lea \& Febiger, 1993: 417-615. 
15. Siegel S - Estadistica no Parametrica. Mexico: Ed. Trillas, 1975 .

16. Vaughan Williams EM - Classification of antiarrhythmic drugs. In: Sandoe E, Flensted-Jensen E, Olesen E - Cardiac Arrhythmias. Sodertalye, Sweden: AB Astra, 1981: 449-72.

17. Josephson ME - Evaluation of antiarrhythmic agents. In: Josephson ME Clinical Cardiac Electrophysiology: Techniques and Interpretations. $2^{\text {nd }} \mathrm{ed}$ Philadelphia: Lea \& Febiger, 1993: 630-82.

18. Marchlinski FE, Buxton AE, Kindawall E, et al - Comparison of individual and combined effects of procainamida and amiodarone in patients with sustained ventricular tachyarrythmias. Circulation 1988; 78: 583-91.

19. Winters SL, Curwin MD, Kan L, et al - Post ESVEM critical pathways to manage ventricular tachycardia: concordance of responses to intravenous procainamide and sotalol at programmed stimulation. PACE 1995; 18: 1736.

20. Távora MZP, De Paola AAV, Silva RMFL, Hara VM, Gondim FAA, Martinez EE - Identificação e ablação por cateter, utilizando radiofrequiência, do circuito de reentrada dos sítios de taquicardia ventricular sustentada em chagásicos. Rev Soc Cardiol Est SP 1995; 5(supl B): 9.

21. Távora MZP, De Paola AAV, Silva RMFL, et al - Influência da função ventricular nas variáveis obtidas no mapeamento endocárdico em portadores de cardiopatia chagásica crônica e taquicardia ventricular sutentada. Arq Bras Cardiol 1993; 61: II-21.

22. Josephson ME, Waxman HL, Cain ME, Gardner MJ, Buxton AE - Ventricular activation during ventricular endocardial pacing. II. Role of pace-mapping to localize origin of ventricular tachycardia. Am J Cardiol 1982; 50: 11-22.

23. Josephson ME, Horowitz LN, Spielman SR, Waxman HL, Greenspan AM - Role of catheter mapping in the preoperative evaluation of ventricular tachycardia. Am J Cardiol 1982; 49: 207-20.

24. Waxman HL, Josephson ME - Ventricular activation during ventricular endocardial pacing: I. Electrocardographic pattern related to the site of pacing. Am J Cardiol 1982; 50: 1-10.

25. El-Sherif N, Meira R, Gough WB, Zeiler RH - Ventricular activation pattern of spontaneous and induced ventricular rhythms in canine one-day-old myocardial infarction. Evidence for focal and reentrant mechanisms. Circ Res 1982; 51: 152-66.

26. El-Sherif N, Meira R, Gough WB, Zeiler RH - Reentrant ventricular arrhythmias in the late myocardial infarction period. Interruption of reentrant circuits by criothermal techniques. Circulation 1983; 68: 644-56.

27. Okumura K, Olshansky B, Henthorn RW, Epstein AE, Plumb VJ, Waldo AL Demonstration of the presence of slow condution during sustained ventricular tachycardia in man: use of trasient entrainment of the tachycardia. Circulation 1987; $75: 369-78$

28. Stevenson WG, Weiss J, Winer I, Wohlgelernter D, Yeatman L - Localization of slow conduction in a ventricular tachycardia circuit: implications for catheter ablation. Am Heart J 1987; 114: 1253-8.

29. Kay GN, Epstein AE, Plumb VJ - Region of slow conduction in sustained ventricular tachycardia: direct endocardial recordings and funcional characterization in humans. J Am Coll Cardiol 1988; 11: 109-16.

30. Morady F, Frank R, Kou WH, et al - Identification and catheter ablation of a zone of slow conduction in the reentrant circuit of ventricular tachycardia in humans. J Am Coll Cardiol 1988; 11: 775-82.

31. Kunze KP, Kuch K-H, Schlüter M - Radiofrequency or direct current for ablation of ventricular tachycardia. J Am Coll Cardiol 1989; 13: 176.

32. Borggrefe M, Chen X, Haverkamp W, et al - Radiofrequency catheter ablation of ventricular tachycardia - single center experience 1986-1993. Circulation 1994; 90: I-558.

33. Kottkamp H, Hindricks G, Chen X, et al - Radiofrequency catheter ablation of sustained ventricular tachycardia in idiopathic dilated cardiomyopathy. Circulation 1995; 92: 1159-68

34. Littmann L, Svenson RH, Gallagher JJ, et al - Funcional role of the epicardium in postinfarction ventricular tachycardia. Observations derived from computerized epicardial activation maping, entraiment, and epicardial laser photoablation. Circulation 1991; 83: 1577-91.

35. Stevenson WG, Weiss J, Winer I, Wohlgelernter D, Yeatman L-Slow conduction in the infarct scar: relevance to the ocurrence, detection and ablation of ventricular reentry circuits resulting from myocardial infarction. Am Heart J 1989; 117: 452-67.

36. Morady F, Kadish A, Rosenheck S, Calkins H, De Buitleir M, Sousa J - Concealed entrainment as a guide for catheter ablation of ventricular tachycardia in patients with prior myocardial infartion. J Am Coll Cardiol 1991; 17: 678-89.

37. Downar E, Kimber S, Harris L, Mickleborough LL, Shaikh N, Parson ID Endocardial mapping of ventricular tachycardia in the intact human ventricle. Evidence for reentry mechanisms. J Am Coll Cardiol 1988; 11: 783-91.

38. Sosa E, Scanavacca M, Martinelli $\mathrm{F}^{\circ} \mathrm{M}$, et al - Técnicas de ablação por cateter (fulguração). Experiência inicial. Arq Bras Cardiol 1988; 50: 253-8.

39. Vattimo ACA - Mapeamento endocárdico em pacientes portadores de cardiopatia chagásica crônica e taquicardia ventricular sustentada. (tese de Mestrado em Cardiologia - Universidade Federal de São Paulo). São Paulo, 1995: 99p. 